\title{
Safety of Tacrolimus in Autoimmune Disease: Results From Post-marketing Surveillance in South Korea
}

\author{
Wan-Hee Yoo, M.D., Ph.D. ${ }^{1}$, Sang-Il Lee, M.D. ${ }^{2}$, Tae-Hwan Kim, M.D., Ph.D. ${ }^{3}$, Jung-Joon Sung, M.D., Ph.D. ${ }^{4}$,

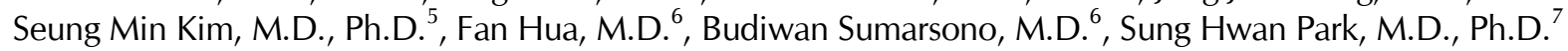 \\ ${ }^{1}$ Division of Rheumatology, Department of Internal Medicine, Jeonbuk National University Medical School, Jeonju, ${ }^{2}$ Department of Internal \\ Medicine, Institute of Health Sciences, Gyeongsang National University School of Medicine, Jinju, ${ }^{3}$ Department of Rheumatology, Hanyang \\ University Hospital for Rheumatic Diseases, Seoul, ${ }^{4}$ Department of Neurology, Seoul National University College of Medicine, Seoul National \\ University Hospital, Seoul, ${ }^{5}$ Department of Neurology, Yonsei University College of Medicine, Seoul, ${ }^{6}$ Astellas Pharma Singapore Pte Ltd., \\ Singapore, 'Division of Rheumatology, Department of Internal Medicine, Seoul St. Mary's Hospital, College of Medicine, The Catholic University \\ of Korea, Seoul, Korea
}

Objective. Tacrolimus, a macrolide immunosuppressant, is approved in Korea for the treatment of rheumatoid arthritis (RA), lupus nephritis ( $\mathrm{LN})$ and myasthenia gravis (MG). We report three prospective post-marketing surveillance studies of tacrolimus conducted in South Korea in these indications. Methods. Studies were conducted according to South Korean Ministry of Food and Drug Safety requirements. Patients were followed up for the duration of the study (up to 4 years) or until treatment discontinuation. Occurrence and likely relationship with tacrolimus of adverse events (AEs), adverse drug reactions (ADRs; defined as AEs where causal relationship to tacrolimus could not be excluded) and serious AEs were recorded. Association of AEs with demographic and medical factors was evaluated by multivariable analysis. Results. The studies included 740 (RA), 307 (LN) and 104 (MG) patients. The incidence of AEs was $12.7 \%$ in RA (64.2\% of AEs potentially related to tacrolimus), $20.9 \%$ (37.8\% potentially related) in LN and $29.8 \%$ (56.8\% potentially related) in MG. The incidence of ADRs was $8.4 \%, 9.8 \%$ and $20.2 \%$, respectively. Serious AEs were reported in $0.7 \%, 7.2 \%$ and $8.7 \%$, respectively. The most common AEs were abdominal pain (RA), pharyngitis (LN) and diarrhea (MG). Unexpected AEs occurred in 3.5\% of patients with RA, $2.9 \%$ in $\mathrm{LN}$ and $8.7 \%$ in MG; no pattern of unexpected AEs was apparent. Multivariable analysis demonstrated that patients with comorbidity had higher probability of experiencing an AE in RA and MG studies. Conclusion. The incidence of AEs and the safety profile of tacrolimus in each indication was consistent with previous reports. (J Rheum Dis 2021;28:202-215)

Key Words. Autoimmune disease, Post-marketing product surveillance, Safety, Tacrolimus

\section{INTRODUCTION}

Tacrolimus is a macrolide immunosuppressant, originally developed to prevent graft rejection in solid organ transplantation. It exerts its immunosuppressive effects by inhibiting calcineurin, which in turn prevents the activation of T-cells [1]. T-cell activation has a key role in the pathogenesis of autoimmune disorders, such as rheumatoid arthritis (RA), lupus nephritis (LN), and myasthenia gravis (MG), making tacrolimus a viable therapeutic op- tion for these conditions. In Korea, tacrolimus is indicated for the 'treatment of some cases of RA where the existing drugs show no effect'. The drug is also approved in Korea for the treatment of LN and MG.

Numerous clinical studies have demonstrated the efficacy and safety of tacrolimus in RA, both as monotherapy [2-5] and, more commonly, in combination with agents such as methotrexate or biologicals [6-13]. A clinical trial of low-dose tacrolimus $(1.5 \mathrm{mg})$ in combination with methotrexate in patients from South Korea demonstrated a

Received : December 28, 2020, Revised : July 5, 2021, Accepted : July 9, 2021

Corresponding to : Sung Hwan Park (iDhttp://orcid.org/0000-0003-1711-2060

Division of Rheumatology, Department of Internal Medicine, Seoul St Mary's Hospital, College of Medicine, The Catholic University of Korea, 222 Banpo-daero, Seocho-gu, Seoul 06591, Korea. E-mail : rapark@catholic.ac.kr

Copyright (c) 2021 by The Korean College of Rheumatology.

This is an Open Access article, which permits unrestricted non-commerical use, distribution, and reproduction in any medium, provided the original work is properly cited. 
43\% response rate according to American College of Rheumatology criteria for a $20 \%$ improvement (ACR20) [13]. A double-blind, randomized trial showed that tacrolimus plus methotrexate was non-inferior to leflunomide plus methotrexate, and was well tolerated in patients from South Korea with moderate-to-severe RA activity [11]. In a retrospective study of patients with RA treated with tacrolimus in a tertiary treatment center in South Korea, $69 \%$ of patients remained on therapy after 4 years, indicating that tacrolimus is effective and well tolerated in this group [12].

In LN, two meta-analyses of tacrolimus concluded that tacrolimus as monotherapy or in combination with mycophenolate mofetil (MMF) improved efficacy compared with cyclophosphamide or azathioprine, whilst a third meta-analysis reported a non-significant trend in favor of tacrolimus [14-16]. In a retrospective multicenter study of ethnically homogenous Korean patients with LN who had failed initial treatment, the complete response rate after 1 year of treatment with tacrolimus plus MMF was $36 \%$ and the overall response rate was $69 \%$ [17]. A steroid-sparing effect was apparent during the study and the combination treatment had a favorable adverse event (AE) profile.

A review of prospective clinical studies of tacrolimus in patients with MG and a meta-analysis have concluded that tacrolimus reduces concomitant steroid dose and improves quantitative MG score and MG activities of daily living $[18,19]$. Tacrolimus appeared to be well tolerated in this patient group $[18,19]$. A multicenter study in South Korea also demonstrated good safety and efficacy for tacrolimus in patients with MG, reporting a reduction in steroid dose and improvement in MG score [20].

As well as evaluating safety and efficacy in clinical trials, it is important to monitor the performance of new drugs or established drugs for new indications under real-world clinical practice conditions. In the present paper, we report the results of three post-marketing surveillance studies of tacrolimus in RA, LN and MG conducted in South Korea. The studies were initiated to provide an assessment of the safety profile of tacrolimus in autoimmune disease indications under real clinical practice conditions.

\section{MATERIALS AND METHODS}

Post-marketing surveillance of tacrolimus was conducted to assess its safety in patients with autoimmune disease. Three prospective surveillance studies were conducted, one in each indication (RA, LN, and MG). Data were collected according to the South Korean Ministry of Food and Drug Safety regulations. Institutional Review Board (IRB) approval was received from each institution, and all patient data were anonymized. The patient consent form was reviewed by each IRB. The safety objectives of the studies were to identify unexpected AEs, AEs occurring under regular treatment conditions, factors considered to influence safety, and new information concerning the safety or mechanism of action of tacrolimus.

\section{Study design}

Each of the studies aimed to enroll more than 300 patients, which is the minimum number required by the South Korean Ministry of Food and Drug Safety for a post-marketing safety surveillance study. All eligible patients attending the participating hospitals and starting treatment for the first time with tacrolimus for RA, LN, or MG were invited to participate. All participating hospitals had experience of using tacrolimus pre-registration. Patients with RA were eligible if sufficient therapeutic response could not be achieved with disease-modifying anti-rheumatic drugs; patients with LN were eligible if they had normal renal function with persistent clinical symptoms of nephritis, despite receiving long-term steroid monotherapy; and patients with MG were eligible if they had no therapeutic response to immunosuppressants or had intolerable side effects.

Patients received treatment according to usual clinical practice in the participating hospital, and were followed up from the start of tacrolimus therapy until they discontinued treatment or the end of the study (up to 4 years). All studies were conducted for 4 years. Patients experiencing an $\mathrm{AE}$ were monitored via visits or telephone contact.

\section{Study outcomes}

The following data were recorded for each patient: demographic information; duration and severity of RA, LN, or MG; concomitant or previous illnesses, including any hepatic or renal impairment; daily dosage of tacrolimus and length of treatment; reason for discontinuation of tacrolimus; use of steroids and immunosuppressants; and use of any other concomitant medication. Severity of disease and presence of comorbidities was based on the investigator's judgement and diagnoses. Hepatic impairment was defined according to the Child-Pugh classi- 
fication and renal impairment according to National Kidney Foundation criteria (G3b or worse) [21,22].

Patients were given diary cards and asked to record AEs. They were also contacted regularly and asked about AEs. The safety analysis recorded the following information: occurrence and duration of new or worsening AEs; severity of AEs (mild, moderate, or severe); treatment of AEs; likely causal relationship to tacrolimus; occurrence of adverse drug reactions (ADRs); and occurrence of serious adverse events (SAEs). Causal relationship to tacrolimus was classified by the investigator according to the South Korean Ministry of Food and Drug Safety criteria as certain, probable/likely, possible, unlikely, conditional/ unclassified, and not assessable/unclassifiable. ADRs were defined according to the South Korean Ministry of Food and Drug Safety definition: any unfavorable and unintended reaction occurring with normal administration of the drug, where a causal relationship to the medicinal product cannot be ruled out. SAEs were defined as causing death or being life-threatening, requiring or prolonging hospitalization, causing persistent or significant disability or incapacity, causing congenital deformity or abnormality, or as another medically significant event.

\section{Statistical analysis}

No formal sample size calculation was performed. The number of AEs and the number and percentage of patients experiencing an $\mathrm{AE}$ were analyzed (frequency analysis). In addition, the association of AEs with demographic and medical factors was evaluated; the dependent variable was the count of AEs (including ADRs) for each patient. Univariable analyses (Pearson's Chi-squared test or Fisher's exact test) were performed to identify any potential predictor variables. Those variables with $\mathrm{p}<0.2$ according to the univariable analysis and variables considered to be clinically relevant were included in subsequent multiple logistic regression analyses, and odds ratios with $95 \%$ confidence intervals were calculated. The SAS statistical analysis software package version 9.2 (SAS Institute, Cary, NC, USA) was used to analyze the data.

\section{RESULTS}

\section{Surveillance study in RA}

The surveillance study was conducted from August 22, 2007 to August 21, 2011. Case record forms for $742 \mathrm{pa}-$ tients were collected from 21 hospitals (60 patients in Year 3 of the study and 682 in Year 4; no data were collected during Years 1 and 2). Two patients were excluded

Table 1. Demographic and clinical characteristics of patients included in the studies

\begin{tabular}{|c|c|c|c|}
\hline Characteristic & RA study $(\mathrm{n}=740)$ & LN study $(n=307)$ & MG study $(n=104)$ \\
\hline \multicolumn{4}{|l|}{ Age $(y r)$} \\
\hline Mean (SD) & $53.7(13.4)$ & $37.3(11.9)$ & $53.5(13.7)$ \\
\hline Range & $7.0 \sim 89.0$ & $13.0 \sim 39.0$ & $21.0 \sim 86.0$ \\
\hline \multicolumn{4}{|l|}{ Age group (yr) } \\
\hline$<20$ & $1(0.1)$ & $15(4.9)$ & 0 \\
\hline 20 to $<40$ & $117(15.8)$ & $169(55.1)$ & $15(14.4)^{*}$ \\
\hline 40 to $<60$ & $350(47.3)$ & $110(35.8)$ & $54(51.9)$ \\
\hline$\geq 60$ & $272(36.8)$ & $13(4.2)$ & $35(33.7)$ \\
\hline Sex, female & $609(82.3)$ & $273(88.9)$ & $60(57.7)$ \\
\hline \multicolumn{4}{|c|}{ Duration of RA, LN, or MG, months } \\
\hline Mean (SD) & $74.2(73.3)$ & $75.8(64.9)$ & $92.0(99.6)$ \\
\hline Median & NA & 60.0 & 42.7 \\
\hline Range & $1 \sim 523$ & $1 \sim 281$ & $0.48 \sim 432$ \\
\hline Concomitant medications & $738(99.7)$ & $307(100.0)$ & $101(97.1)$ \\
\hline Current comorbidity & $409(55.3)$ & $307(100.0)$ & 82 (78.9) \\
\hline Previous illness & NR & $90(29.3)$ & $28(26.9)$ \\
\hline Current illness & NR & $307(100.0)$ & $82(78.9)$ \\
\hline Hepatic impairment & $30(4.1)$ & $11(3.6)$ & $2(1.9)$ \\
\hline Renal impairment & $10(1.4)$ & $108(35.2)$ & 0 \\
\hline
\end{tabular}

Data expressed as $\mathrm{n}(\%)$ patients unless otherwise stated. LN: lupus nephritis, MG: myasthenia gravis, NR: not recorded, RA: rheumatoid arthritis, SD: standard deviation, NA: not available. ${ }^{*} \geq 19$ to $<40$ years. 
from the safety analysis because tacrolimus had been administered before the start of the study. Of the 740 patients included, there was one child $(0.1 \%)$ and 160 $(21.6 \%)$ patients $\geq 65$ years of age; most patients $(82.3 \%)$ were female (Table 1). Complete age distribution is shown in Table 1. The mean duration of RA was 74.2 months, and most patients $(72.8 \%)$ were classified as having RA of moderate severity. Ten (1.4\%) patients had renal impairment and $30(4.1 \%)$ patients had hepatic impairment (Table 1). Further characteristics are shown in Supplementary Tables 1 and 2.

A total of 521 (70.4\%) patients received tacrolimus for longer than 24 weeks. The mean (standard deviation [SD]) daily dosage of tacrolimus was 1.50 (0.46) mg; $57.3 \%$ received $<1.5 \mathrm{mg} /$ day, $42.6 \%$ received $1.5 \sim 3$ $\mathrm{mg} /$ day, and $0.1 \%$ received $>3 \mathrm{mg} /$ day (the maximum approved dose is $3 \mathrm{mg} /$ day). The mean (SD) duration of treatment was 203 (100) days; 479 patients $(64.7 \%)$ received treatment for 168 364 days; 88 (11.9\%) for $<84$ days; 131 (17.7\%) for 84 167 days; and 42 (5.7\%) for $\geq$ 365 days. Of the 88 patients who received fewer than 84 days of treatment, 37 discontinued due to lack of efficacy, 33 because of AEs, one because of symptom improvement, seven for other reasons, and 10 were lost to follow-up.

A total of 123 AEs was reported in 94 (12.7\%) patients, including 78 ADRs in 62 (8.4\%) patients (Table 2). Gastrointestinal disorders were the main class of event reported in $\geq 0.5 \%$ of patients (Table 2 ). Abdominal pain was the only AE that occurred in $\geq 1 \%$ of patients, reported in eight (1.1\%) patients. Most AEs (61.8\%) were considered to be of mild severity, $32.5 \%$ of moderate severity, and $5.7 \%$ were severe (Table 2). Severe AEs were elevated erythrocyte sedimentation rate, elevated levels of C-reactive protein, muscle pain, palpitations, surgical intervention (two events), and myocardial infarction. Causal relationship with tacrolimus was assessed as certain for $4.1 \%$ of events, probable/likely for $18.7 \%$, possible for $33.3 \%$, unlikely for $35.8 \%$, conditional/unclassified for $3.3 \%$, and not assessable/unclassifiable for $4.9 \%$ (Table 2). Of all the AEs, gastrointestinal abnormalities were most commonly considered to be related to tacrolimus, with 25 out of 35 (71.4\%) gastrointestinal AEs being considered certainly, probably or possibly related. A total of 60 out of 123 AEs (48.8\%) resulted in discontinuation of tacrolimus (Table 2). The only AEs leading to discontinuation in more than two patients were abdominal pain (six patients), nausea (five patients), and rash (four patients).

Nine SAEs were reported in five $(0.7 \%)$ patients. One patient experienced deterioration in physical condition, asthenia, gastric ulcer and pruritus; one patient experienced two surgical interventions; and one patient each experienced cervical cancer, myocardial infarction, and worsening RA (Table 2). The worsening RA event was classified as a serious ADR.

Of 160 patients $\geq 65$ years of age, 24 (15.0\%) patients reported 31 AEs and 16 (10.0\%) reported 20 ADRs. The pattern of AEs and ADRs was similar to the overall population (Supplementary Table 3). Ten patients in the study had renal impairment, of whom five reported six AEs: hiccups, constipation, abdominal pain, residual urine, elevated urine creatinine, and headache. Thirty patients had hepatic impairment, five of whom reported five AEs: elevated serum glutamic-oxaloacetic transaminase, elevated serum glutamic pyruvic transaminase, abdominal pain, peripheral edema, and headache.

A total of 33 unexpected AEs occurred in 26 patients (3.5\%), of which 17 in 14 patients were considered as ADRs: elevated C-reactive protein (three patients); elevated erythrocyte sedimentation rate, facial edema and unspecified gastrointestinal disorder (two patients each); and xerostomia, hiccups, infectious arthritis, arthritis, vaginitis, vaginal bleeding, dermatitis and worsening RA (one patient each).

\section{1) Analysis of factors associated with AEs}

The univariable analysis showed that patients' demographic characteristics were not associated with the incidence of AEs (Supplementary Table 4). Of the medical characteristics analyzed, the incidence of AEs was significantly higher in patients with any complication or comorbidity $(\mathrm{p}=0.002)$ and in patients with renal impairment $(\mathrm{p}=0.005)$ (Supplementary Table 4$)$. The multivariable analysis confirmed that patients with a complication or comorbidity had a higher probability of experiencing an $\mathrm{AE}$ (Figure 1).

\section{Surveillance study in LN}

Between November 9, 2009 and November 8, 2013, case record forms for 314 patients were collected from 23 centers. Seven patients were excluded from the safety analysis: one because the patient received tacrolimus for an unapproved indication and six because precautions for use of tacrolimus were not adhered to. Of the 307 patients included, $10(3.3 \%)$ were $<19$ years of age and four 
Table 2. Adverse events and adverse drug reactions reported in the RA study

\begin{tabular}{|c|c|c|}
\hline \multirow{2}{*}{ Event } & \multicolumn{2}{|c|}{ No. patients or events (\%) } \\
\hline & AEs & ADRs \\
\hline Patients with AE or ADR ( $n=740$ patients) & $94(12.7)$ & $62(8.4)$ \\
\hline AEs potentially related to tacrolimus* $(n=123$ AEs) & $79(64.2)$ & NR \\
\hline AEs leading to discontinuation of tacrolimus ${ }^{\dagger}(n=123 \mathrm{AEs})$ & $60(48.8)$ & NR \\
\hline \multicolumn{3}{|l|}{ Severity $(n=123$ AEs) } \\
\hline Mild & $76(61.8)$ & NR \\
\hline Moderate & $40(32.5)$ & NR \\
\hline Severe & $7(5.7)$ & NR \\
\hline \multicolumn{3}{|l|}{ AE or ADR occurring in $\geq 0.5 \%$ of patients ( $n=740$ patients) } \\
\hline \multicolumn{3}{|l|}{ Gastrointestinal disorders } \\
\hline Abdominal pain & $8(1.1)$ & $7(1.0)$ \\
\hline Dyspepsia & $5(0.7)$ & $5(0.7)$ \\
\hline Nausea & $5(0.7)$ & $5(0.7)$ \\
\hline Diarrhea & $4(0.5)$ & $4(0.5)$ \\
\hline \multicolumn{3}{|l|}{ General disorders and administration site conditions } \\
\hline ESR increased & $6(0.8)$ & $2(0.3)$ \\
\hline \multicolumn{3}{|l|}{ Hepatobiliary disorders } \\
\hline SGPT increased & $4(0.5)$ & $3(0.4)$ \\
\hline \multicolumn{3}{|l|}{ Investigations } \\
\hline CRP increased & $5(0.7)$ & $3(0.4)$ \\
\hline \multicolumn{3}{|l|}{ Renal and urinary disorders } \\
\hline Face edema & $4(0.5)$ & $2(0.3)$ \\
\hline \multicolumn{3}{|l|}{ Skin and subcutaneous tissue disorders } \\
\hline Rash & $4(0.5)$ & $4(0.5)$ \\
\hline SAEs ( $\mathrm{n}=740$ patients) & $5(0.7)$ & $1(0.1)$ \\
\hline \multicolumn{3}{|l|}{ Cardiac disorders } \\
\hline Myocardial infarction & $1(0.1)$ & 0 \\
\hline \multicolumn{3}{|l|}{ Gastrointestinal disorders } \\
\hline Gastric ulcer & $1(0.1)$ & 0 \\
\hline \multicolumn{3}{|l|}{ General disorders and administration site conditions } \\
\hline Deterioration in physical condition & $1(0.1)$ & 0 \\
\hline Asthenia & $1(0.1)$ & 0 \\
\hline \multicolumn{3}{|l|}{ Musculoskeletal and connective tissue disorders } \\
\hline Worsening RA & $1(0.1)$ & $1(0.1)$ \\
\hline \multicolumn{3}{|l|}{ Neoplasms } \\
\hline Cervical carcinoma & $1(0.1)$ & 0 \\
\hline \multicolumn{3}{|l|}{ Skin and subcutaneous tissue disorders } \\
\hline Pruritus & $1(0.1)$ & 0 \\
\hline \multicolumn{3}{|l|}{ Surgical and medical procedures } \\
\hline Surgical intervention & $1(0.1)$ & 0 \\
\hline
\end{tabular}

ADR: adverse drug reaction, AE: adverse event, CRP: C-reactive protein, ESR: erythrocyte sedimentation rate, NR: not reported, RA: rheumatoid arthritis, SAE: serious adverse event, SGPT: serum glutamic-pyruvic transaminase. *Includes AEs classified as certainly, probably/likely, or possibly related to tacrolimus, or those recorded as conditional/unclassified or not assessable/unclassifiable. ${ }^{\dagger}$ AEs leading to discontinuation were abdominal pain, indigestion, diarrhea, nausea, constipation, unspecified gastrointestinal disorder, gastroenterocolitis, xerostomia, vomiting, gastric ulcer, elevated erythrocyte sedimentation rate, peripheral edema, asthenia, edema, deterioration in physical condition, pharyngitis, cough, hepatosis, cholelithiasis, elevated C-reactive protein, dizziness, hypoesthesia, headache, muscle pain, infectious arthritis, arthralgia, rash, itchiness, dermatitis, facial edema, panhematopenia, palpitation, high pulse rate, loss of appetite, hyperglycemia, dazzled vision, vaginitis, vaginal bleeding, and residual urine. $123 \mathrm{AEs}$ were reported in 94 patients. 


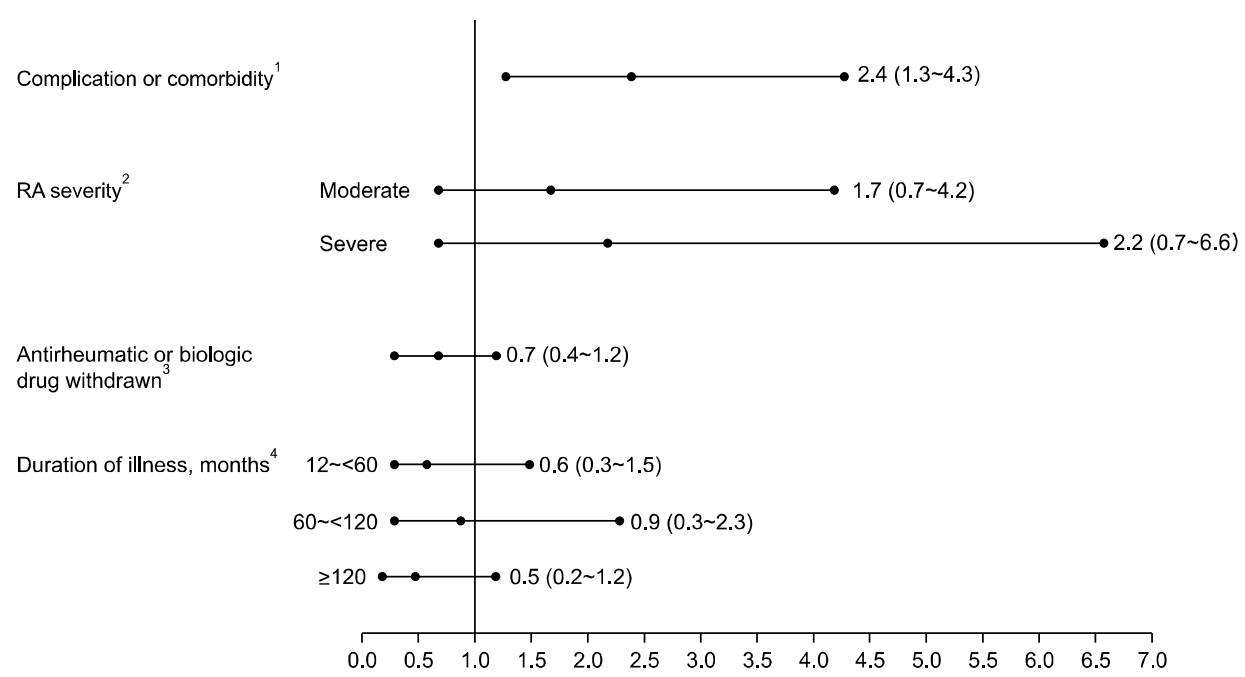

Figure 1. Odds ratios and $95 \% \mathrm{Cls}$ for the probability of experiencing an adverse event in the multivariable analysis according to the medical characteristics of the patient (RA study). 1. Presence versus absence of complication or comorbidity; 2 . Moderate or severe RA versus mild RA; 3. Presence versus absence of any antirheumatic or biologic drug withdrawn before tacrolimus administration; 4. Duration of illness: period shown versus $<12$ months. $\mathrm{Cl}$ : confidence interval, RA: rheumatoid arthritis, RA: rheumatoid arthritis.

(1.3\%) were $\geq 65$ years of age; most patients $(88.9 \%)$ were female (Table 1). Complete age distribution is shown in Table 1. The median duration of $\mathrm{LN}$ was 60.0 months, and $61.6 \%$ were classified as having LN of moderate severity. A total of 108 (35.2\%) patients had renal impairment, and 11 (3.6\%) had hepatic impairment (Table 1). Further characteristics are shown in Supplementary Tables 1 and 5 .

The mean (SD) daily dose of tacrolimus was 2.1 (1.0) $\mathrm{mg}$ and the mean (SD) total dose was $664.6(568.6) \mathrm{mg}$ (range $0.5 \sim 3,014 \mathrm{mg}$ ). The mean (SD) duration of treatment was 309 (221) days and the median was 279 days. A total of 66 (21.5\%) patients discontinued tacrolimus during the study, 21 (6.8\%) because of an AE, 31 (10.1\%) because of lack of efficacy or no response, four (1.3\%) because of improvement in symptoms, and 10 (3.3\%) for other reasons.

A total of 98 AEs occurred in 64 (20.8\%) patients, including 37 ADRs in 30 (9.8\%) patients (Table 3). Pharyngitis was the most common AE and ADR. Most AEs $(68 ; 69.4 \%)$ were considered to be of mild severity, 21 (21.4\%) of moderate severity, and 9 (9.2\%) were severe (Table 3). A total of 37 out of 98 AEs (37.8\%) were assessed as being certainly, probably, or possibly related to tacrolimus, or as conditional/unclassified or not assessable/unclassifiable (Table 3). Of the 98 AEs, 29 resulted in discontinuation of tacrolimus (Table 3). All AEs leading to discontinuation occurred in one or two pa- tients, with the exception of albuminuria, which occurred in three patients.

Twenty-nine SAEs were reported in 22 (7.2\%) patients, with seven patients experiencing two SAEs: herpes zoster and gastroenteritis; varicella and appendicitis; leg pain and pneumonia; azotemia and abdominal pain; nausea and increased alkaline phosphatase; insomnia and diarrhea; cystitis and abdominal pain (Table 3).Ten events in nine patients $(2.9 \%)$ were classified as a serious ADR. Twelve SAEs were classified as mild, 12 as moderate, and five as severe. Twenty-five events resolved, two developed further, and one led to death (azotemia [elevated creatinine], considered unlikely to be related to tacrolimus; this patient also experienced abdominal pain classified as a SAE, which resolved).

Four of the ten participants $<19$ years of age experienced eight AEs (two ADRs in one participant), while no AEs or ADRs occurred in the four participants $\geq 65$ years of age. Of 108 participants with renal impairment, $47 \mathrm{AEs}$ occurred in 30 participants (27.8\%) and 15 ADRs in 11 participants (10.2\%). Of 11 participants with hepatic impairment, nine AEs occurred in five participants (45.5\%) and eight ADRs in five participants (45.5\%).

Eleven unexpected AEs occurred in 9 (2.9\%) patients: two reports of an autoantibody reaction in a single patient, one report in a single patient of varicella and appendicitis, and one report in a single patient each of aggravated systemic lupus erythematosus (SLE), verruca, 
Table 3. Adverse events and adverse drug reactions reported in the LN study

\begin{tabular}{|c|c|c|}
\hline \multirow{2}{*}{ Event } & \multicolumn{2}{|c|}{ No. patients or events (\%) } \\
\hline & AEs & ADRs \\
\hline Patients with AE or ADR ( $n=307$ patients) & $64(20.9)$ & $30(9.8)$ \\
\hline AEs considered potentially related to tacrolimus $*(n=98 \mathrm{AEs})$ & $37(37.8)$ & NR \\
\hline AEs leading to discontinuation of tacrolimus ${ }^{\dagger}(\mathrm{n}=98 \mathrm{AEs})$ & $29(29.6)$ & NR \\
\hline \multicolumn{3}{|l|}{ Severity $(n=98$ AEs) } \\
\hline Mild & $68(69.4)$ & NR \\
\hline Moderate & $21(21.4)$ & NR \\
\hline Severe & $9(9.2)$ & NR \\
\hline \multicolumn{3}{|l|}{ AEs occurring in $\geq 0.5 \%$ of patients ( $n=307$ patients) } \\
\hline \multicolumn{3}{|l|}{ Gastrointestinal disorders } \\
\hline Nausea & $5(1.6)$ & $2(0.7)$ \\
\hline Diarrhea & $4(1.3)$ & $1(0.3)$ \\
\hline Dyspepsia & $4(1.3)$ & $1(0.3)$ \\
\hline Abdominal pain & $2(0.7)$ & $1(0.3)$ \\
\hline \multicolumn{3}{|l|}{ General disorders and administration site conditions } \\
\hline Edema & $2(0.7)$ & 0 \\
\hline Leg pain & $2(0.7)$ & 0 \\
\hline Fever & $2(0.7)$ & $1(0.3)$ \\
\hline Cellulitis & $2(0.7)$ & $1(0.3)$ \\
\hline \multicolumn{3}{|l|}{ Infections and infestations } \\
\hline Herpes zoster & $2(0.7)$ & $1(0.3)$ \\
\hline \multicolumn{3}{|l|}{ Metabolism and nutrition disorders } \\
\hline Hypercholesterolemia & $3(1.0)$ & 0 \\
\hline \multicolumn{3}{|l|}{ Nervous system disorders } \\
\hline Headache & $3(1.0)$ & $2(0.7)$ \\
\hline Seizure & $2(0.7)$ & $1(0.3)$ \\
\hline \multicolumn{3}{|l|}{ Renal and urinary disorders } \\
\hline Albuminuria & $3(1.0)$ & $2(0.7)$ \\
\hline Azotemia & $3(1.0)$ & $2(0.7)$ \\
\hline Decreased creatinine clearance & $3(1.0)$ & $3(1.0)$ \\
\hline \multicolumn{3}{|l|}{ Respiratory, thoracic and mediastinal disorders } \\
\hline Pharyngitis & $7(2.3)$ & $4(1.3)$ \\
\hline Upper respiratory tract infection & $2(0.7)$ & 0 \\
\hline \multicolumn{3}{|l|}{ Vascular disorders } \\
\hline Hypertension & $2(0.7)$ & $2(0.7)$ \\
\hline SAEs $(\mathrm{n}=307$ patients $)$ & $22(7.2)$ & $9(2.9)$ \\
\hline \multicolumn{3}{|l|}{ Blood and lymphatic system disorders } \\
\hline Thrombocytopenia & $1(0.3)$ & $1(0.3)$ \\
\hline \multicolumn{3}{|l|}{ Gastrointestinal disorders } \\
\hline Abdominal pain & $2(0.7)$ & $1(0.3)$ \\
\hline Diarrhea & $1(0.3)$ & 0 \\
\hline Nausea & $1(0.3)$ & 0 \\
\hline Gastroenteritis & $1(0.3)$ & 0 \\
\hline Appendicitis & $1(0.3)$ & 0 \\
\hline \multicolumn{3}{|l|}{ General disorders and administration site conditions } \\
\hline Leg pain & $1(0.3)$ & 0 \\
\hline Edema & $1(0.3)$ & 0 \\
\hline Fever & $1(0.3)$ & 0 \\
\hline Pain & $1(0.3)$ & 0 \\
\hline Cellulitis & $1(0.3)$ & $1(0.3)$ \\
\hline \multicolumn{3}{|l|}{ Hepatobiliary disorders } \\
\hline Cholecystitis & $1(0.3)$ & 0 \\
\hline
\end{tabular}


Table 3. Continued

\begin{tabular}{|c|c|c|}
\hline \multirow{2}{*}{ Event } & \multicolumn{2}{|c|}{ No. patients or events (\%) } \\
\hline & AEs & ADRs \\
\hline \multicolumn{3}{|l|}{ Infections and infestations } \\
\hline Herpes zoster & $2(0.7)$ & $1(0.3)$ \\
\hline Infection & $1(0.3)$ & $1(0.3)$ \\
\hline Varicella & $1(0.3)$ & 0 \\
\hline \multicolumn{3}{|l|}{ Metabolism and nutrition disorders } \\
\hline Hyperglycemia & $1(0.3)$ & $1(0.3)$ \\
\hline Aggravated diabetes mellitus & $1(0.3)$ & 0 \\
\hline Increased alkaline phosphatase & $1(0.3)$ & 0 \\
\hline \multicolumn{3}{|c|}{ Musculoskeletal and connective tissue disorders } \\
\hline Aggravated SLE & $1(0.3)$ & 0 \\
\hline \multicolumn{3}{|l|}{ Nervous system disorders } \\
\hline Seizure & $1(0.3)$ & 0 \\
\hline \multicolumn{3}{|l|}{ Psychiatric disorders } \\
\hline Insomnia & $1(0.3)$ & 0 \\
\hline \multicolumn{3}{|l|}{ Renal and urinary disorders } \\
\hline Azotemia & $2(0.7)$ & $1(0.3)$ \\
\hline Cystitis & $1(0.3)$ & 0 \\
\hline Albuminuria & $1(0.3)$ & $1(0.3)$ \\
\hline Decreased creatinine clearance & $1(0.3)$ & $1(0.3)$ \\
\hline \multicolumn{3}{|c|}{ Respiratory, thoracic and mediastinal disorders } \\
\hline Pneumonia & $1(0.3)$ & $1(0.3)$ \\
\hline
\end{tabular}

ADR: adverse drug reaction, AE: adverse event, LN: lupus nephritis, NR: not reported, SAE: serious adverse event, SLE: systemic lupus erythematosus. *Includes AEs classified as certainly, probably/likely, or possibly related to tacrolimus, or thoserecorded as conditional/unclassified or not assessable/unclassifiable. ${ }^{\dagger}$ AEs leading to discontinuation were: nausea, diarrhea, abdominal pain, vomiting, albuminuria, azotemia, decreased creatinine clearance, hematuria, pneumonia, fever, pain, hyperglycemia, headache, dizziness, eczema, alopecia, unspecified infection, auto-antibody response, cellulitis, hypertension, and thrombocytopenia. 98 AEs were reported in 64 patients.

eczema, cholecystitis, pyelonephritis, amenorrhea, and hyperpyrexia. Of these, the autoantibody reaction, verru$\mathrm{ca}$, and pyelonephritis were classified as ADRs. Cholecystitis, varicella, appendicitis, and aggravated SLE were considered as SAEs.

\section{1) Analysis of factors associated with AEs}

No statistically significant association between any AE and any demographic factor was identified (Supplementary Table 6). The univariable analysis identified a statistically significant association between the incidence of AEs and renal impairment, with AEs occurring in 30/108 (27.8\%) of patients with renal impairment and 34/199 (17.1\%) without renal impairment $(\mathrm{p}=0.028)$ (Supplementary Table 6). No association was seen with concomitant or previous illness, hepatic or cardiac impairment, severity of LN, history of treatment with immunosuppressants, reason for prescription of tacrolimus, or concomitant medication. The multivariable analysis demonstrated no statistically significant association between any medical characteristic and the occurrence of AEs (Figure 2).

\section{Surveillance study in MG}

Case record forms for 194 patients were collected from 15 centers between June 10, 2015 and June 9, 2019. Ninety patients were excluded from the safety analysis, three who were lost to follow up and 87 because they did not fulfil the eligibility criteria of having no therapeutic response or intolerable side effects to immunosuppressant therapy taken before the study. Of the 104 patients included, 22 (21.2\%) patients were $\geq 65$ years of age and $57.7 \%$ of patients were female (Table 1). Complete age distribution is shown in Table 1. The median duration of MG was 42.7 months. The severity of MG was classified according to the Myasthenia Gravis Foundation of America (MGFA) clinical classification: Class 1 19.2\%, Class 2a 29.8\%, Class 2b 19.2\%, Class 3a 10.6\%, Class 3b 7.7\%, Class 4a 0\%, Class 4b 1.0\%, and unknown $10.6 \%$. 


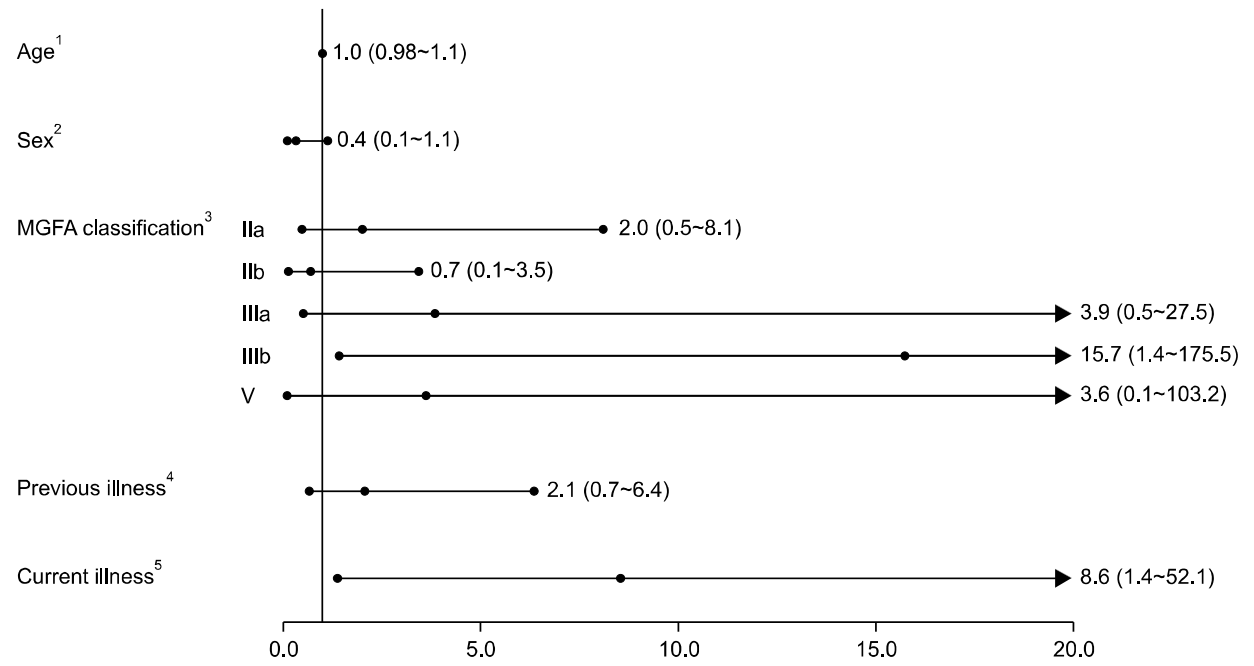

Figure 2. Odds ratios and 95\% Cls for the probability of experiencing an adverse event in the multivariable analysis according to the medical characteristics of the patient (LN study). 1. Presence of previous illness versus unknown; 2 . Absence of previous illness versus uncertain; 3. Presence versus absence of renal impairment; 4. Mild LN versus severe LN; 5 . Moderate $\mathrm{LN}$ versus severe $\mathrm{LN}$. $\mathrm{Cl}$ : confidence interval, $\mathrm{LN}$ : lupus nephritis.
No patients had renal impairment and two (1.9\%) had hepatic impairment (Table 1). Further characteristics are shown in Supplementary Tables 1 and 7.

The mean (SD) daily dose of tacrolimus was 3.0 (0.0) $\mathrm{mg}$ and the total mean (SD) dose was 628.6 (246.2) mg (range 84.0 1704.0). The mean (SD) duration of treatment was 210 (82) days and the median was 211 days. Fourteen $(13.5 \%)$ patients discontinued tacrolimus during the study, 10 because of an $\mathrm{AE}$, one because of lack of efficacy, and three for other reasons.

A total of $31(29.8 \%)$ patients experienced 44 AEs, including 25 ADRs in 21 (20.2\%) patients (Table 4). Diarrhea and alopecia were the most common AEs and ADRs. Most AEs (30 [68.2\%]) were considered to be mild, 11 (25.0\%) moderate, and $3(6.8 \%)$ severe. Just over half of the AEs (25 [56.8\%]) were considered potentially related to tacrolimus (Table 4). Fourteen of the 44 AEs $(31.8 \%)$ led to discontinuation of tacrolimus; only diarrhea and alopecia occurred in more than two patients (three patients each).

Nine SAEs were reported in nine $(8.7 \%)$ patients (Table 4), including four events classified as a serious ADR (Table 4). Three of the four ADRs were considered possibly related to tacrolimus (diabetes mellitus, hyperglycemia, and cytomegalovirus infection); the fourth ADR (cardiac arrest) resulted in death and was classified as conditional/unclassified. The patient who died had a number of comorbidities: myofascial pain syndrome, sinusitis, subclinical hypothyroidism, osteopenia and a cyst on the lung. Unexpected AEs occurred in nine (8.7\%) patients: heartburn (two patients), epigastric disorder, retinopathy, leiomyoma of uterus, cardiac arrest, lumbar pain, hoarseness, and common cold. Of these, heartburn (one patient), epigastric discomfort, and cardiac arrest were classified as ADRs.

\section{1) Analysis of factors associated with AEs}

The univariable analysis found several demographic and medical characteristics associated with the occurrence of AEs (Supplementary Table 8 ). The incidence of AEs was higher in women than men $(p=0.026)$, and in patients with previous or current illness $(p=0.006$ and 0.017 , respectively). The incidence of AEs also varied according to the MGFA clinical classification $(p=0.029)$; AEs occurred in $5 / 20(25 \%)$ patients in Class $1,10 / 31(32 \%)$ in Class 2a, 5/20 (25\%) in Class 2b, 4/11 (36\%) in Class 3a, 6/8 $(75 \%)$ in Class $3 \mathrm{~b}, 0 / 1$ in Class $4 \mathrm{~b}, 1 / 2$ (50\%) in Class 5 , and $0 / 11$ whose Class was unknown (no patients were in Class 4a) (Supplementary Table 8). Owing to the low patient numbers in Classes 4 and 5, the pattern of AE incidence by MGFA Class cannot be interpreted. There was no significant association with the other factors analyzed, including duration of MG, concomitant therapy, age $\geq 65$ years, or hepatic or renal impairment (Supplementary Table 8). The multivariable analysis indicated that there was a significantly higher incidence of AEs in patients in MGFA Class $3 \mathrm{~b}$ versus patients in Class $1(\mathrm{p}=0.025)$, and in patients with current illness versus patients with no illness $(\mathrm{p}=0.020)$ (Figure 3$)$.

\section{DISCUSSION}

While clinical trials have provided invaluable data regarding the safety and efficacy of tacrolimus in autoimmune diseases, it is important to monitor the performance of tacrolimus under real-world clinical practice con- 
Table 4. Adverse events and adverse drug reactions reported in the MG study

\begin{tabular}{|c|c|c|}
\hline \multirow{2}{*}{ Event } & \multicolumn{2}{|c|}{ No. patients or events (\%) } \\
\hline & AEs & ADRs \\
\hline Patients with AE or ADR ( $n=104$ patients) & $31(29.8)$ & $21(20.2)$ \\
\hline AEs considered potentially related to tacrolimus* $(n=44$ AEs) & $25(56.8)$ & NR \\
\hline AEs leading to discontinuation of tacrolimus ${ }^{\dagger}(\mathrm{n}=44 \mathrm{AEs})$ & $14(31.8)$ & NR \\
\hline \multicolumn{3}{|l|}{ Severity $(n=44$ AEs) } \\
\hline Mild & $30(68.2)$ & NR \\
\hline Moderate & $11(25.0)$ & NR \\
\hline Severe & $3(6.8)$ & NR \\
\hline \multicolumn{3}{|l|}{ All AEs $^{\ddagger}(n=104$ patients $)$} \\
\hline \multicolumn{3}{|l|}{ Blood and lymphatic system disorders } \\
\hline Anemia & $1(1.0)$ & 0 \\
\hline \multicolumn{3}{|l|}{ Cardiac disorders } \\
\hline Cardiac arrest & $1(1.0)$ & $1(1.0)$ \\
\hline \multicolumn{3}{|l|}{ Eye disorders } \\
\hline Retinopathy & $1(1.0)$ & 0 \\
\hline \multicolumn{3}{|l|}{ Gastrointestinal disorders } \\
\hline Diarrhea & $6(5.8)$ & $6(5.8)$ \\
\hline Heartburn & $2(1.9)$ & $1(1.0)$ \\
\hline Dyspepsia & $2(1.9)$ & $2(1.9)$ \\
\hline Epigastric discomfort & $1(1.0)$ & $1(1.0)$ \\
\hline Dysphagia & $1(1.0)$ & 0 \\
\hline Nausea & $1(1.0)$ & $1(1.0)$ \\
\hline Bowel motility disorder & $1(1.0)$ & $1(1.0)$ \\
\hline \multicolumn{3}{|l|}{ General disorders and administration site conditions } \\
\hline Chest pain & $1(1.0)$ & 0 \\
\hline Leg pain & $1(1.0)$ & 0 \\
\hline Lumbar pain & $1(1.0)$ & 0 \\
\hline \multicolumn{3}{|l|}{ Infections and infestations } \\
\hline Herpes zoster & $2(1.9)$ & $2(1.9)$ \\
\hline Cytomegalovirus infection & $1(1.0)$ & $1(1.0)$ \\
\hline \multicolumn{3}{|l|}{ Metabolism and nutrition disorders } \\
\hline Hypercholesterolemia & $1(1.0)$ & 0 \\
\hline Hyperglycemia & $1(1.0)$ & $1(1.0)$ \\
\hline Diabetes mellitus & $1(1.0)$ & $1(1.0)$ \\
\hline Weight decrease & $1(1.0)$ & 0 \\
\hline \multicolumn{3}{|l|}{ Musculoskeletal and connective tissue disorders } \\
\hline Myalgia & $1(1.0)$ & 0 \\
\hline \multicolumn{3}{|l|}{ Neoplasms } \\
\hline Uterine leiomyoma & $1(1.0)$ & 0 \\
\hline \multicolumn{3}{|l|}{ Nervous system disorders } \\
\hline Headache & $2(1.9)$ & $2(1.9)$ \\
\hline Hoarseness & $1(1.0)$ & 0 \\
\hline \multicolumn{3}{|l|}{ Renal and urinary disorders } \\
\hline Urinary tract infection & $1(1.0)$ & 0 \\
\hline Urinary incontinence & $1(1.0)$ & 0 \\
\hline \multicolumn{3}{|l|}{ Respiratory, thoracic and mediastinal disorders } \\
\hline Common cold & $1(1.0)$ & 0 \\
\hline Dyspnea & $1(1.0)$ & 0 \\
\hline \multicolumn{3}{|l|}{ Skin and subcutaneous tissue disorders } \\
\hline Alopecia & $5(4.8)$ & $5(4.8)$ \\
\hline Increased sweating & $1(1.0)$ & 0 \\
\hline Hairiness & $1(1.0)$ & 0 \\
\hline
\end{tabular}


Table 4. Continued

\begin{tabular}{|c|c|c|}
\hline \multirow{2}{*}{ Event } & \multicolumn{2}{|c|}{ No. patients or events (\%) } \\
\hline & AEs & ADRs \\
\hline SAEs ( $n=104$ patients $)$ & $9(8.7)$ & $4(3.9)$ \\
\hline \multicolumn{3}{|l|}{ Cardiac disorders } \\
\hline Cardiac arrest & $1(1.0)$ & $1(1.0)$ \\
\hline \multicolumn{3}{|l|}{ Gastrointestinal disorders } \\
\hline Dysphagia & $1(1.0)$ & 0 \\
\hline \multicolumn{3}{|c|}{ General disorders and administration site conditions } \\
\hline Chest pain & $1(1.0)$ & 0 \\
\hline Leg pain & $1(1.0)$ & 0 \\
\hline Lumbar pain & $1(1.0)$ & 0 \\
\hline \multicolumn{3}{|l|}{ Infections and infestations } \\
\hline Cytomegalovirus infection & $1(1.0)$ & $1(1.0)$ \\
\hline \multicolumn{3}{|c|}{ Metabolism and nutrition disorders } \\
\hline Hyperglycemia & $1(1.0)$ & $1(1.0)$ \\
\hline Diabetes mellitus & $1(1.0)$ & $1(1.0)$ \\
\hline \multicolumn{3}{|l|}{ Neoplasms } \\
\hline Uterine leiomyoma & $1(1.0)$ & 0 \\
\hline
\end{tabular}

ADR: adverse drug reaction, AE: adverse event, MG: myasthenia gravis, NR: not reported, SAE: serious adverse event. *Includes AEs classified as certainly, probably/likely, or possibly related to tacrolimus, or those recorded as conditional/unclassified or not assessable/unclassifiable. ${ }^{\dagger}$ AEs leading to discontinuation were: diarrhea, heartburn, dyspepsia, nausea, alopecia, hyperglycemia, herpes zoster, headache, and cardiac arrest. ${ }^{\ddagger}$ All AEs reported are shown. 44 AEs were reported in 31 patients.

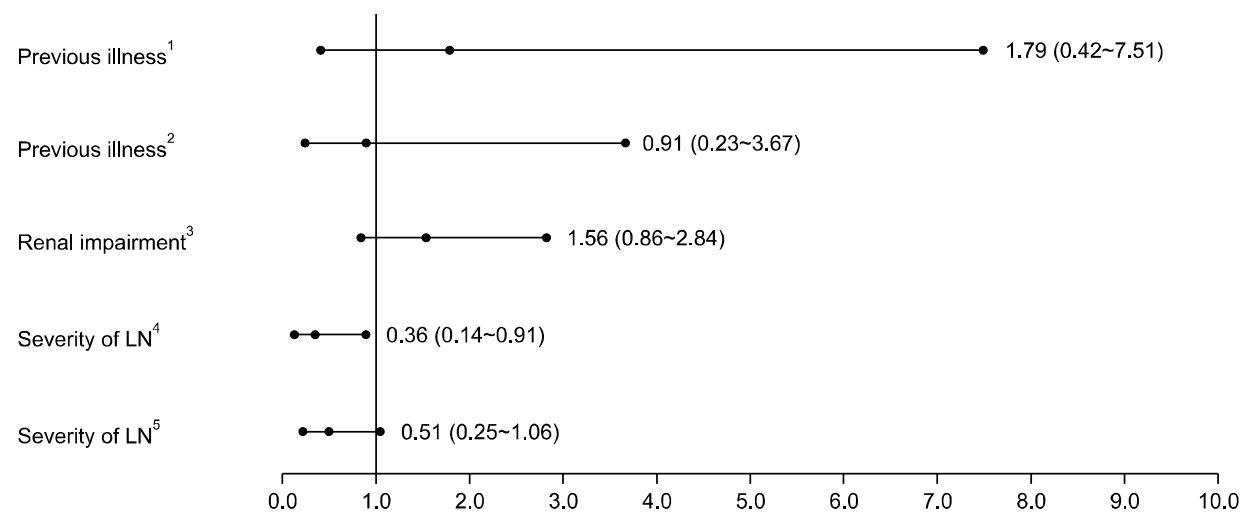

Figure 3. Odds ratios and $95 \% \mathrm{Cls}$ for the probability of experiencing an adverse event in the multivariable analysis according to the medical characteristics of the patient (MG study). 1. Continuous variable; 2. Male versus female; 3. Stated Class versus Class 1; 4. Presence versus absence of previous illness; 5. Presence versus absence of current illness. Cl: confidence interval, MG: myasthenia gravis, MGFA: Myasthenia Gravis Foundation of America.

ditions, such as through post-marketing surveillance. In these post-marketing surveillance studies of tacrolimus in RA, LN and MG, the incidence of AEs and ADRs was not markedly different to that observed in other studies. Few unexpected AEs were reported; of the unexpected AEs that were considered as ADRs (suspected causal relationship to tacrolimus), each individual event occurred in fewer than $1 \%$ of patients.

The incidence of AEs was $12.7 \%$ in RA, $20.9 \%$ in LN, and $29.8 \%$ in MG. SAEs were reported in $0.7 \%$ of patients with RA, $7.2 \%$ with $\mathrm{LN}$, and $8.7 \%$ with MG. The incidence of ADRs followed a similar pattern to AEs and was low considering the long surveillance period (approximately $10 \%$ in patients with RA and LN, and $20 \%$ in patients with MG). Differences in the incidence of AEs might be explained by differences in tacrolimus dose and duration of exposure in the three indications; indeed, the mean daily dose was $1.5 \mathrm{mg}$ in RA, $2.1 \mathrm{mg}$ in $\mathrm{LN}$ and 
3.0 mg in MG. Another explanation could be the different underlying diseases. As such, the safety profile of tacrolimus should be considered in each disease separately in terms of underlying disease activity, patient demographic characteristics, comorbidities and treatment protocols. For patients with RA, the incidence of ADRs was comparable with that reported in a Japanese post-marketing surveillance study of patients receiving add-on tacrolimus over 24 weeks following an inadequate response to biologic DMARDs $[23,24]$. An interim analysis of this study in 172 patients reported that $10.5 \%$ of patients experienced an ADR, whilst the final analysis in 624 patients reported an incidence of $15.1 \%$ [23,24]. By contrast, in patients with $\mathrm{LN}$, the incidence of ADRs was lower than cited in the recent Japanese post-marketing surveillance TRUST study that evaluated patients who were initiated and maintained on tacrolimus therapy over 5 years ( $9.8 \%$ vs. $57.0 \%$, respectively) [25]. This is possibly driven by the difference in size of the populations studied (1,355 vs. 307). Other plausible explanations might be the disparity in penetration of the surveillance systems, treatment protocols, length of exposure to tacrolimus, population characteristics and overall follow-up duration. For patients with MG, while there are no published data from similar post-marketing surveillance studies, the incidence of AEs and SAEs was comparable with a previously reported 4-year retrospective Chinese study (24.7\% and $7.2 \%$, respectively) [26].

Although the AE and ADR profiles differed between the three autoimmune diseases assessed, the most common events cited were generally consistent with previous reports. For example, the most common AEs and ADRs reported for patients with RA were gastrointestinal disorders-particularly abdominal pain-which is in line with findings from the Japanese post-marketing surveillance study by Ishida et al. [23]. and clinical studies, including those involving Korean patients [11,13,27]. Pharyngitis was the most common AE and ADR in patients with $\mathrm{LN}$, consistent with the post-marketing surveillance TRUST study in which the most common ADRs included nasopharyngitis [25]. Diarrhea and alopecia were the most common AEs and ADRs in patients with MG, and hyperglycemia was reported as an SAE in one patient. Gastrointestinal disturbances accounted for approximately one-third of all ADRs reported in a multicenter study in Korean patients with MG receiving tacrolimus [20], and were common AEs in a retrospective study of 160 Korean patients [28]. Co-occurrence of MG and alopecia has been previously reported [29]. Notably, the incidences of AEs of concern in solid organ transplant patients receiving tacrolimus (namely hypertension, tremor, diabetes and increased creatinine level) were low in these post-marketing surveillance studies of autoimmune diseases. This might be because the dose of tacrolimus used for transplant patients is higher than for RA, LN and MG, and the patient characteristics are different in each disease. The lack of AEs of concern seen in transplant patients is encouraging for clinicians, but highlights that the AE profile for tacrolimus differs depending on the clinical indication for its use.

Because of the narrow therapeutic index of tacrolimus, more dose adjustments are required during the first weeks of treatment than during long-term use. It might, therefore, be expected that patients will experience relatively more AEs and ADRs during short-term use compared with long-term use when a steady dose and blood drug level are established. On the other hand, long-term use might be associated with a higher incidence of opportunistic infections as a result of a compromised immune function. However, the low incidence of infections reported here suggests that long-term use of tacrolimus was not associated with an unacceptably increased risk of infection in patients with RA, LN and MG.

In these post-marketing surveillance studies, the nature and incidence of ADRs were as expected from the package insert. However, 33 unexpected AEs were reported in RA patients, of which 17 were considered ADRs: elevated C-reactive protein, elevated erythrocyte sedimentation rate, facial edema, unspecified gastrointestinal disorder, xerostomia, hiccups, infectious arthritis, arthritis, vaginitis, vaginal bleeding, dermatitis and worsening RA. Eleven unexpected AEs were reported in patients with LN, of which three (autoantibody reaction, verruca and pyelonephritis) were considered ADRs, and nine unexpected AEs were reported in patients with MG (heartburn, epigastric discomfort and cardiac arrest were considered ADRs). The unexpected AEs and ADRs could be attributed to patients' underlying disease or other confounding factors. Further research is required in this area. This highlights the importance of continued post-marketing surveillance to raise awareness among clinicians regarding the AEs that might be experienced by their patients.

Results of the multivariable logistic regression analysis in patients with RA showed that the presence of a complication or comorbidity was associated with a higher in- 
cidence of AEs compared with their absence. Similarly, in patients with MG, the presence of current illness was associated with a higher incidence of AEs compared with its absence, as was the presence of Class $3 \mathrm{~b}$ illness compared with Class 1 . These findings are expected, as AEs may develop due to underlying conditions during the administration of tacrolimus. However, no statistically significant association between background medical factors and the incidence of AEs was identified in the LN study. In patients with MG, the incidence of AEs was higher in women than in men in the univariable analysis $(p=$ 0.026), although there was no significant difference in the multivariable analysis. No other studies of tacrolimus in RA, LN or MG have compared the incidence of AEs between sexes; however, a study of patients in the US MG patient registry receiving prednisone found that women reported AEs and intolerable AEs more frequently than men (95\% vs. $81 \%$ and $77 \%$ vs. $50 \%$, respectively) [30]. Whether these findings are the result of women experiencing more AEs than men, being more willing than men to report AEs, or for other reasons, is unclear.

These analyses were associated with the limitations typical of post-marketing surveillance studies. For example, no data were available regarding the number or characteristics of patients who declined to participate or were lost to follow-up. Although the aim was to collect data from 300 patients in each study, only 104 patients with MG were included. The analysis of demographic and medical factors affecting safety outcomes did not adjust for the length of exposure to tacrolimus. Furthermore, as data were collected from Korean patients, the safety of tacrolimus in autoimmune diseases should be confirmed in other patient populations. Despite their limitations, these post-marketing surveillance studies included a relatively large number of patients and provide useful long-term data regarding the safety of tacrolimus in autoimmune diseases under real-life clinical practice conditions.

\section{CONCLUSION}

In these post-marketing surveillance studies, the overall incidence of AEs was low, but several unexpected AEs were identified. This highlights the need to routinely perform drug surveillance in patients with autoimmune disease receiving tacrolimus therapy. However, results were aligned with previous and the most recent safety data on tacrolimus in these indications.

\section{ACKNOWLEDGMENTS}

The studies were sponsored by Astellas Pharma South Korea. Mary Greenacre $\mathrm{PhD}$ assisted in drafting the manuscript under the direction of the authors for Cello Health MedErgy, and provided editorial support throughout its development.

\section{CONFLICT OF INTEREST}

All authors report non-financial support from Astellas Pharma, Inc., during the conduct of the study. FH and BS are employees of Astellas Pharma Singapore Pte Ltd., Singapore.

\section{AUTHOR CONTRIBUTIONS}

Writing - reviewing and editing: W.H.Y., S.I.L., T.H.K., J.J.S., S.M.K., F.H., B.S., S.H.P. Investigation: W.H.Y., S.I.L., T.H.K., J.J.S., S.M.K., S.H.P. Conceptualization: S.H.P. Methodology: S.H.P.

\section{DATA SHARING STATEMENT}

Researchers may request access to anonymized participant level data, trial level data and protocols from Astellas sponsored clinical trials at www.clinicalstudydatarequest. com. For the Astellas criteria on data sharing see: https:// clinicalstudydatarequest.com/Study-Sponsors/Study-Sp onsors-Astellas.aspx.

\section{SUPPLEMENTARY DATA}

Supplementary data can be found with this article online at https://doi.org/10.4078/jrd.2021.28.4.202.

\section{REFERENCES}

1. Pallet N, Fernández-Ramos AA, Loriot MA. Impact of immunosuppressive drugs on the metabolism of $\mathrm{T}$ cells. Int Rev Cell Mol Biol 2018;341:169-200.

2. Kawai S, Hashimoto H, Kondo H, Murayama T, Kiuchi T, Abe T. Comparison of tacrolimus and mizoribine in a randomized, double-blind controlled study in patients with rheumatoid arthritis. J Rheumatol 2006;33:2153-61.

3. Kondo H, Abe T, Hashimoto H, Uchida S, Irimajiri S, Hara $\mathrm{M}$, et al. Efficacy and safety of tacrolimus (FK506) in treatment of rheumatoid arthritis: a randomized, double blind, placebo controlled dose-finding study. J Rheumatol 2004; 31:243-51. 
4. Yocum DE, Furst DE, Kaine JL, Baldassare AR, Stevenson JT, Borton MA, et al. Efficacy and safety of tacrolimus in patients with rheumatoid arthritis: a double-blind trial. Arthritis Rheum 2003;48:3328-37.

5. Furst DE, Saag K, Fleischmann MR, Sherrer Y, Block JA, Schnitzer T, et al. Efficacy of tacrolimus in rheumatoid arthritis patients who have been treated unsuccessfully with methotrexate: a six-month, double-blind, randomized, doseranging study. Arthritis Rheum 2002;46:2020-8.

6. Kitahama M, Nakajima A, Inoue E, Taniguchi A, Momohara $S$, Yamanaka $\mathrm{H}$. Efficacy of adjunct tacrolimus treatment in patients with rheumatoid arthritis with inadequate responses to methotrexate. Mod Rheumatol 2013;23:788-93.

7. Ogasawara M, Tamura N, Kageyama M, Onuma S, Kusaoi $\mathrm{M}$, Toyama $\mathrm{S}$, et al. Single-center, retrospective analysis of efficacy and safety of tacrolimus as a second-line DMARD in combination therapy and the risk factors contributing to adverse events in 115 patients with rheumatoid arthritis. Clin Rheumatol 2012;31:251-7.

8. Tanaka Y, Kawai S, Takeuchi T, Yamamoto K, Miyasaka N. Prevention of joint destruction by tacrolimus in patients with early rheumatoid arthritis: a post hoc analysis of a double-blind, randomized, placebo-controlled study. Mod Rheumatol 2013;23:1045-52.

9. Fujibayashi T, Takahashi N, Kida D, Kaneko A, Hirano Y, Fukaya N, et al. Comparison of efficacy and safety of tacrolimus and methotrexate in combination with abatacept in patients with rheumatoid arthritis; a retrospective observational study in the TBC Registry. Mod Rheumatol 2015;25:825-30.

10. Kaneshiro S, Ebina K, Hirao M, Tsuboi H, Nishikawa M, Nampei A, et al. The efficacy and safety of additional administration of tacrolimus in patients with rheumatoid arthritis who showed an inadequate response to tocilizumab. Mod Rheumatol 2017;27:42-9.

11. Shin K, Baek HJ, Kang YM, Cha HS, Kang SW, Park SH, et al. Efficacy and safety of add-on tacrolimus versus leflunomide in rheumatoid arthritis patients with inadequate response to methotrexate. Int J Rheum Dis 2019;22:1115-22.

12. Park EY, Lee SG, Park EK, Koo DW, Park JH, Kim GT, et al. Drug survival and the associated predictors in South Korean patients with rheumatoid arthritis receiving tacrolimus. Korean J Intern Med 2018;33:193-202.

13. Lee WS, Lee SI, Lee MS, Kim SI, Lee SS, Yoo WH. Efficacy and safety of low-dose tacrolimus for active rheumatoid arthritis with an inadequate response to methotrexate. Korean J Intern Med 2016;31:779-87.

14. Hannah J, Casian A, D'Cruz D. Tacrolimus use in lupus nephritis: a systematic review and meta-analysis. Autoimmun Rev 2016;15:93-101.

15. Kraaij T, Bredewold OW, Trompet S, Huizinga TW, Rabelink TJ, de Craen AJ, et al. TAC-TIC use of tacrolimus-based regimens in lupus nephritis. Lupus Sci Med 2016;3:e000169.

16. Lee YH, Song GG. Comparative efficacy and safety of tacrolimus, mycophenolate mofetil, azathioprine, and cyclophosphamide as maintenance therapy for lupus nephritis: a Bayesian network meta-analysis of randomized controlled trials. Z Rheumatol 2017;76:904-12.
17. Park DJ, Kang JH, Lee KE, Bae SC, Chung WT, Choe JY, et al. Efficacy and safety of mycophenolate mofetil and tacrolimus combination therapy in patients with lupus nephritis: a nationwide multicentre study. Clin Exp Rheumatol 2019; 37:89-96.

18. Cruz JL, Wolff ML, Vanderman AJ, Brown JN. The emerging role of tacrolimus in myasthenia gravis. Ther Adv Neurol Disord 2015;8:92-103.

19. Wang L, Xi J, Zhang S, Wu H, Zhou L, Lu J, et al. Effectiveness and safety of tacrolimus therapy for myasthenia gravis: a single arm meta-analysis. J Clin Neurosci 2019;63:160-7.

20. Ahn SW, Joo IS, Kim BJ, Sung JJ, Kang SY, Oh J, et al. A multicenter prospective observational study on the safety and efficacy of tacrolimus in patients with myasthenia gravis. J Neurol Sci 2017;379:271-5.

21. Pugh RN, Murray-Lyon IM, Dawson JL, Pietroni MC, Williams R. Transection of the oesophagus for bleeding oesophageal varices. Br J Surg 1973;60:646-9.

22. National Kidney Foundation. How to classify CKD [Internet]. New York (NY): National Kidney Foundation [cited 2020 Jul 8]. Available from: https://www.kidney.org/professionals/ explore-your-knowledge/how-to-classify-ckd.

23. Ishida K, Shiraki K, Yoshiyasu T. Evaluation of the safety and effectiveness of add-on tacrolimus in patients with rheumatoid arthritis who failed to show an adequate response to biological DMARDs: the interim results of a specific drug use-results survey of tacrolimus. Drugs R D 2015;15:307-17.

24. Takeuchi T, Ishida K, Shiraki K, Yoshiyasu T. Safety and effectiveness of tacrolimus add-on therapy for rheumatoid arthritis patients without an adequate response to biological disease-modifying anti-rheumatic drugs (DMARDs): post-marketing surveillance in Japan. Mod Rheumatol 2018;28:48-57.

25. Takeuchi T, Wakasugi N, Uno S, Makino H. Long-term safety and effectiveness of tacrolimus in patients with lupus nephritis: 5-year interim postmarketing surveillance study in Japan (TRUST). J Rheumatol 2021;48:74-81.

26. Tao X, Wang W, Jing F, Wang Z, Chen Y, Wei D, et al. Long-term efficacy and side effects of low-dose tacrolimus for the treatment of Myasthenia Gravis. Neurol Sci 2017; 38:325-30.

27. Kawai S, Yamamoto K. Safety of tacrolimus, an immunosuppressive agent, in the treatment of rheumatoid arthritis in elderly patients. Rheumatology (Oxford) 2006;45: 441-4.

28. Kim YH, Shin HY, Kim SM. Long-term safety and efficacy of tacrolimus in myasthenia gravis. Yonsei Med J 2019; 60:633-9.

29. Suzuki S, Shimoda M, Kawamura M, Sato H, Nogawa S, Tanaka K, et al. Myasthenia gravis accompanied by alopecia areata: clinical and immunogenetic aspects. Eur J Neurol 2005; 12:566-70.

30. Lee I, Kaminski HJ, McPherson T, Feese M, Cutter G. Gender differences in prednisone adverse effects: survey result from the MG registry. Neurol Neuroimmunol Neuroinflamm 2018;5:e507. 